\title{
Leptomeningeal Metastasis as the First Presentation of Lung Adenocarcinoma
}

\author{
Sena Guler ${ }^{1 *}$, Hatem Hakan Selcuk ${ }^{2}$, Fulya Eren ${ }^{1}$, Evsen Apaydin ${ }^{3}$ and Aysun Soysal ${ }^{1}$
}

${ }^{1}$ Department of Neurology, Bakırköy Prof. Dr. Mazhar Osman Training and Research Hospital for Psychiatric and Neurological Diseases, , Istanbul, Turkey ${ }^{2}$ Department of Neuroradiology, Bakırköy Dr. Sadi Konuk Training and Research Hospital, Istanbul, Turkey

${ }^{3}$ Department of Pathology, Istanbul Faculty of Medicine, Istanbul University, Istanbul, Turkey

\begin{abstract}
Leptomeningeal metastasis is a late and devastating complication of malignancies. Due to wide range of neurological signs and symptoms, it may be difficult to diagnose when there is no systemic complaints. Here, we present a case of a 41-year-old male with sensory loss as the first manifestation of lung adenocarcinoma.
\end{abstract}

Keywords: Lung adenocarcinoma; Leptomeningeal metastasis; TTF-1

\section{Case Report}

A 41-year-old male patient admitted to emergency department with the history of sensory loss, starting from right arm and spreading to right leg, that lasted for 35 to 40 minutes. According to the history taken from his relatives, he had difficulty in understanding and making long sentences in last few days. He was misdiagnosed as focal epilepsy and started antiepileptic therapy in another hospital and there was no improvement in his complaints. There was no known chronic disease history and no medication at the time of admission to the hospital.

His neurological examination revealed no abnormalities apart from diplopia while looking at the left side. He did not have any systemic symptom and any abnormality on physical examination. Brain Magnetic Resonance Imaging (MRI) with contrast showed diffuse leptomeningeal thickening and gadolinium enhancement, prominent in left frontal region, enhancement in cerebellar folia and the third, fifth, seventh and eighth cranial nerves bilaterally (Figures 1A-C). A Computed Tomography (CT) of the brain with contrast showed subarachnoid contrast enhancement in cerebral sulci in the same region (Figure 1D). Due to leptomeningeal involvement, lumbar puncture was performed. In Cerebrospinal Fluid (CSF) examination, 25 leukocytes were found with the protein of $128 \mathrm{mg} / \mathrm{dL}$ and $25 \mathrm{~cm} \mathrm{H}_{2} \mathrm{O}$ of CSF opening pressure. Glucose level in CSF was $19 \mathrm{mg} / \mathrm{dL}$ while the level of blood glucose was $84 \mathrm{mg} / \mathrm{dL}$. The patient was hospitalized for further investigation for infectious causes, lymphoma, sarcoidosis and metastasis. Antituberculosis treatment as INH $300 \mathrm{mg} /$ day, rifampicin $600 \mathrm{mg} /$ day, pyrazinamide $2000 \mathrm{mg} /$ day, ethambutol $1500 \mathrm{mg} /$ day was started as tuberculosis could not be ruled out.

In the first week of his follow-up, he showed disorganized behaviors. Because differential diagnosis included metastasis, lumbar puncture was repeated for three times for the pathological examination of atypical cells in CSF. There was a gradual increase in the number of leukocytes $(0,35,140)$ and protein level $(106.9,168,315 \mathrm{mg} / \mathrm{dL})$ (Figure 2). Given the patient's disease progression and considering his history of growing and inhaling Reishi mushroom (ganoderma lucidum), antituberculosis treatment was stopped at the sixth day of treatment, empirical meropenem 6 gr/day and antifungal treatment with amphotericin B $450 \mathrm{mg} /$ day was started at the same time, although there was no spore or hyphea formation in direct microscopic examination. CSF cultures for bacteria, tuberculosis and fungi in Saboraund dextrose agar and Brucella agglutination test were performed and all was negative. To rule out any kind of epileptic activity and encephalitis, electroencephalography (EEG) was done and found to be normal.
The pathological examination revealed atypical epithelial cells, strongly suggesting malignancy in all CSF samples (Figure 3A). Immunohistochemically, these cells were positive for Cytokeratin-7, Thyroid transcription factor-1 (TTF-1) and Napsin-A (Figures 3B-D), which indicates metastasis of lung adenocarcinoma.

The chest X-ray showed radiopaque density secondary to atelectasis in the right lower lobe (Figure 4A), which prompted a chest CT scan showing a nodular lesion with the size of $31 \times 26 \mathrm{~mm}$ in right lower lobe (Figure 4B). At this point, bronchoscopy was planned to reach to the intrapulmonary lesion. Biopsy was taken from the right middle lobe, revealing TTF-1 positive adenocarcinoma, consistent with the findings in CSF.

Accordingly, Positron Emission Tomography (PET)/CT were performed to stage the cancer and showed increased fluorodeoxyglucose (FDG) uptake in right middle lobe, vertebral column, pelvic bones, ribs and scapula in both sides. Brain and abdominal region could not be evaluated because of motion artifact. Meanwhile, due to amphotericin B therapy, his liver enzymes were elevated. An abdominal ultrasonography was done, showing hyperechogenic nodule in the liver. Further investigation was planned with abdominal MRI with contrast showing liver metastases as well. After the etiology had been revealed, antibacterial and antifungal treatment was stopped at the seventh day of treatment. He transferred to an oncology department of another hospital for his definitive treatment.

\section{Discussion}

Leptomeningeal metastasis is the infiltration of leptomeninges by malignant cells originating from an extrameningeal tumor site. The incidence of leptomeningel metastasis in patients with solid tumors is $5 \%$, whereas it is $20 \%$ in autopsy series [1,2]. Comparing all solid tumors metastasing to leptomeninges, breast cancer, lung cancer and melanoma are the most common ones [1,3,4]. Among lung cancer,

*Corresponding author: Dr. Sena Guler, Bakırköy Prof. Dr. Mazhar Osman Training and Research Hospital for Psychiatric and Neurological Diseases, Neurology, Istanbul, Turkey ; E-mail: aksoysena13@gmail.com

Received November 22, 2018; Accepted December 12, 2018; Published December 17, 2018

Citation: Guler S, Selcuk HH, Eren F, Apaydin E, Soysal A (2018) Leptomeningeal Metastasis as the First Presentation of Lung Adenocarcinoma. J Neurol Neurophysiol 9: 475. doi:10.4172/2155-9562.1000475

Copyright: $\odot 2018$ Guler S, et al. This is an open-access article distributed unde the terms of the Creative Commons Attribution License, which permits unrestricted use, distribution, and reproduction in any medium, provided the original author and source are credited. 
Citation: Guler S, Selcuk HH, Eren F, Apaydin E, Soysal A (2018) Leptomeningeal Metastasis as the First Presentation of Lung Adenocarcinoma. J Neurol Neurophysiol 9: 475. doi:10.4172/2155-9562.1000475

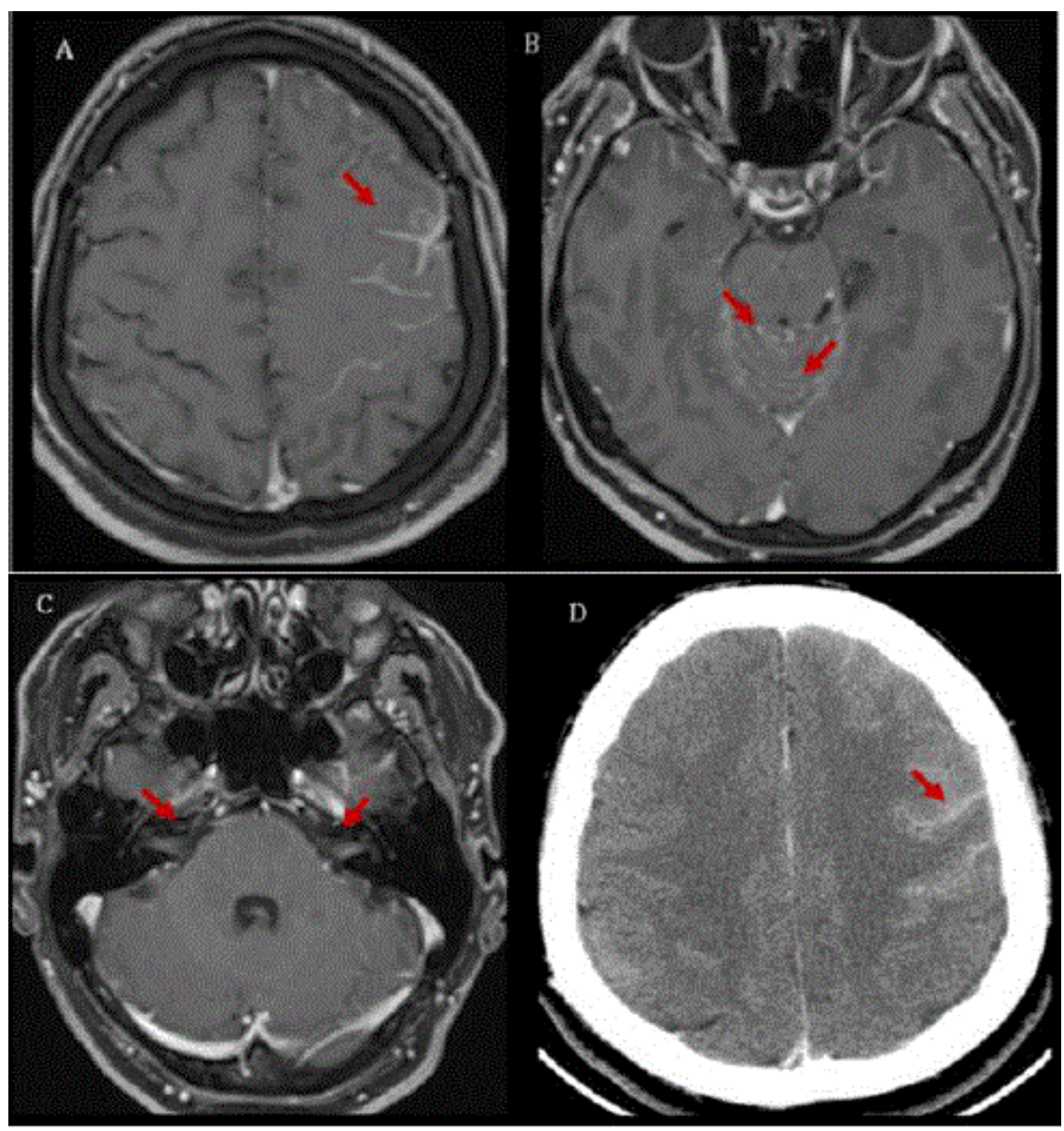

Figure 1: (A) Brain MRI with contrast showing leptomeningeal thickening and gadolinium enhancement in left frontal region, (B) Enhancement in cerebellar folia, (C) Bilateral the seventh and eighth cranial nerves, (D) Head CT with contrast showing subarachnoid contrast enhancement in cerebral sulci.

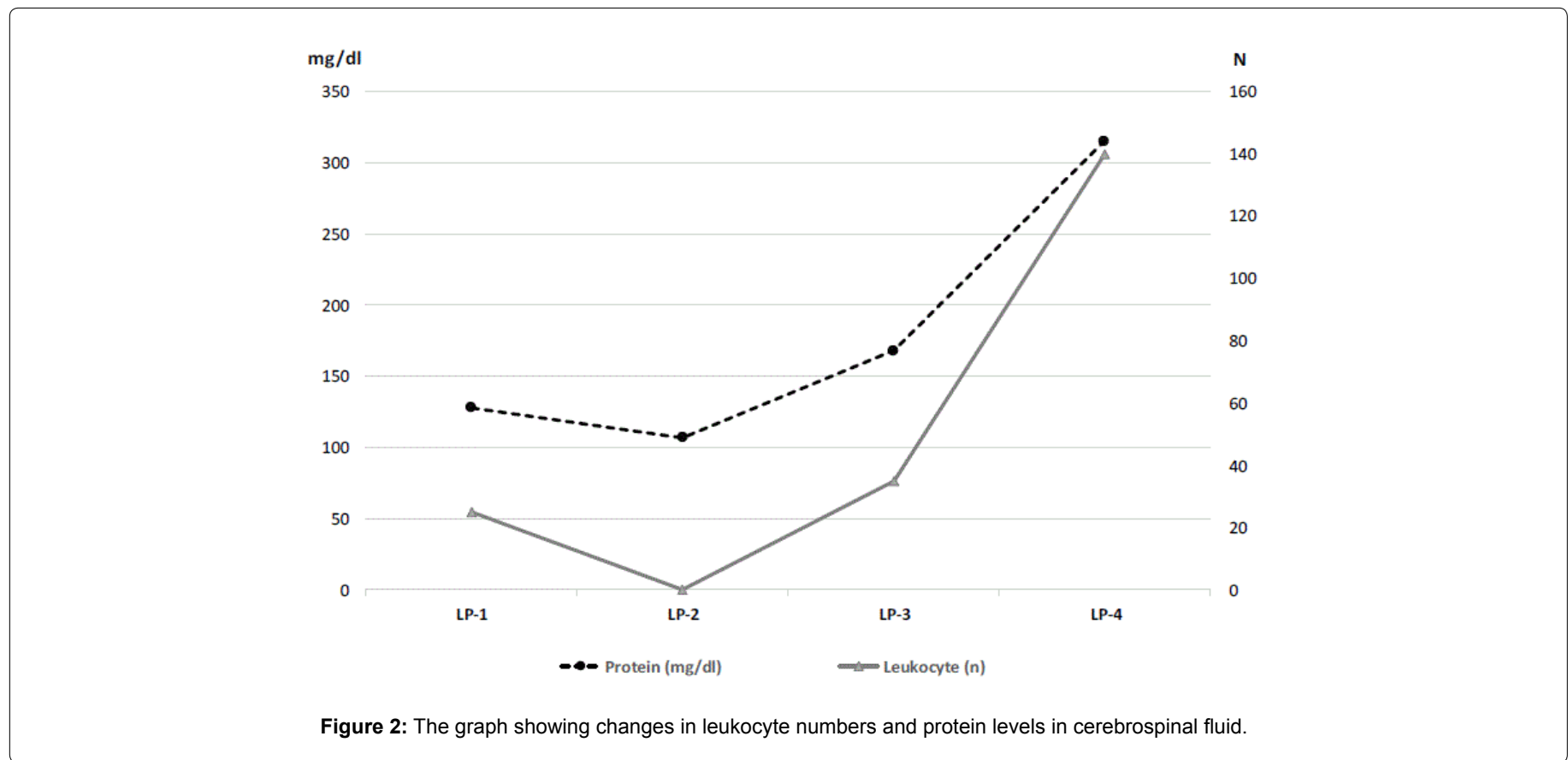


Citation: Guler S, Selcuk HH, Eren F, Apaydin E, Soysal A (2018) Leptomeningeal Metastasis as the First Presentation of Lung Adenocarcinoma. J Neurol Neurophysiol 9: 475. doi:10.4172/2155-9562.1000475
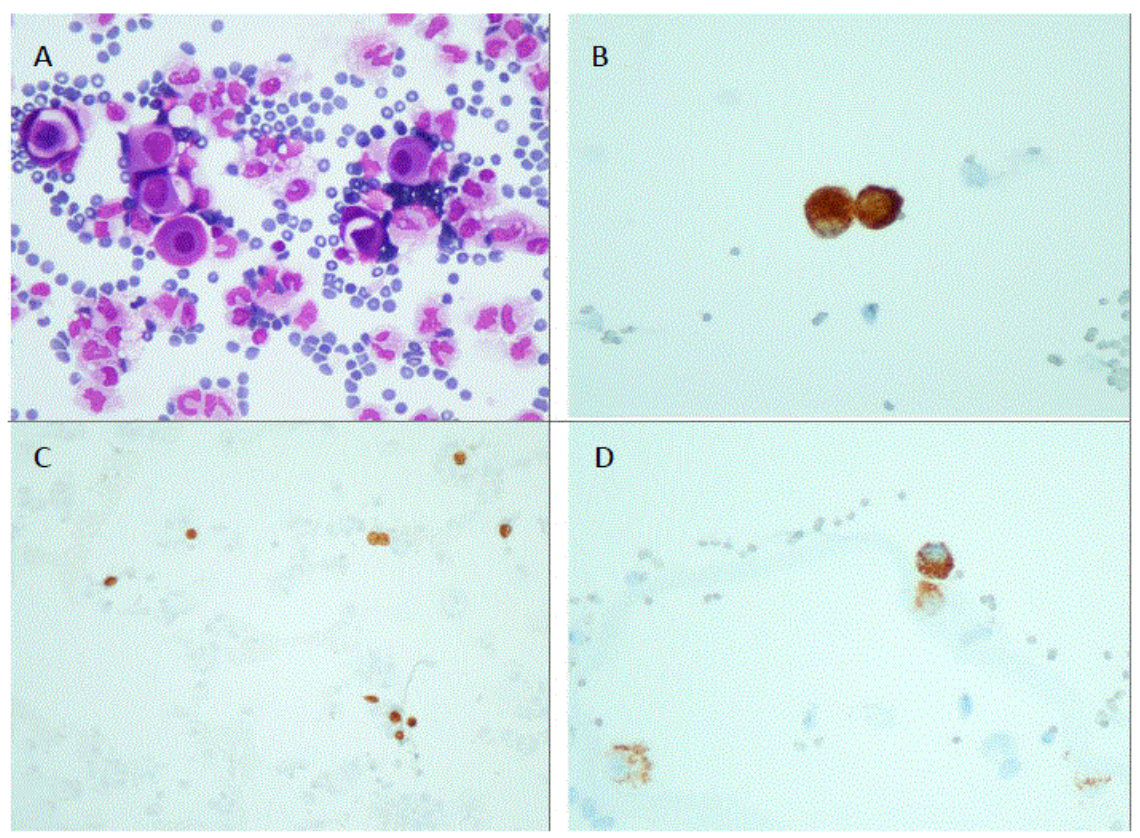

Figure 3: (A) Small groups of epithelial cells with mild atypia in cerebrospinal fluid (May Grünwald Giemsa, $x 200$ ), (B) Tumor cells showing Cytokeratin-7 immunopositivity (anti-Cytokeratin-7 antibody, x400), (C) Nuclear positivity for TTF-1 in tumor cells (anti-TTF-1 antibody, x100), (D) Granular-cytoplasmic immunopositivity for Napsin-A (anti-Napsin-A antibody, x400).

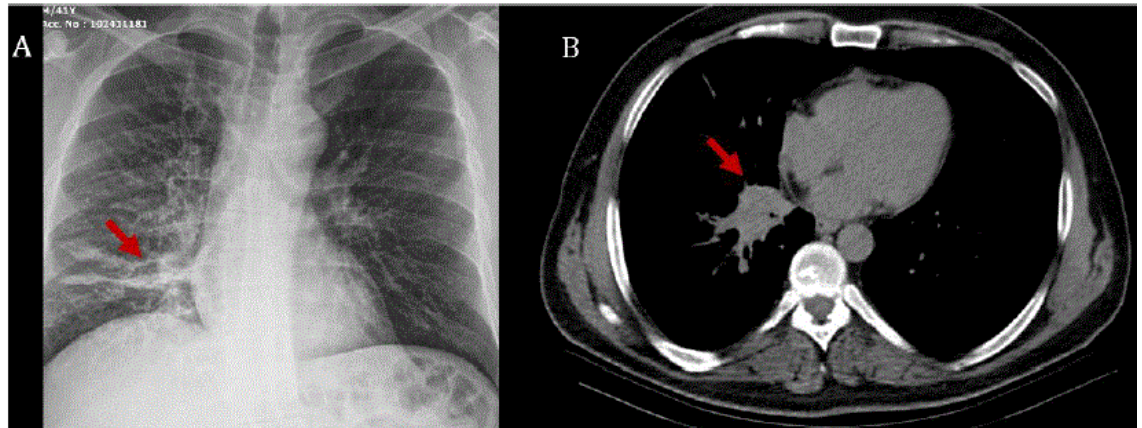

Figure 4: (A) The chest X-ray showing radiopaque density secondary to atelectasis in the right lower lobe, (B) chest CT scan showing a nodular lesion with soft tissue density in right lower lobe, mediastinal window.

$92 \%$ are non-small cell lung carcinoma whereas $8 \%$ are small cell lung carcinoma [5]. Due to better results of new systemic chemotherapeutic agents, the risk of brain metastasis in non-small cell lung carcinoma is increasing, up to $40 \%$ [6]. Malignant cells may invade leptomeninges through hematogenous spread, iatrogenic spread, direct spread from brain parenchyma or endoneural/ perineural an perivascular lymphatic spread as seen in breast and lung cancer. Cancer cells fill Virchow-Robin spaces, and cause blood-brain barrier dysfunction, cranial and spinal nerve dmyelination, axonal degeneration and infarction in infiltrated areas $[1,4]$.

Without any doubt, earlier diagnosis has great importance to prevent fixed nervous system dysfunction. The most commonly seen neurological symptoms can be divided into three domains of neurological function; cerebral hemispheres as headache and change in mental status, cranial nerves as diplopia and facial paresis and spinal cord as lower motor weakness, paresthesia and radiculopathy [1]. Although leptomeningeal metastasis is a late complication of malignancies, it can also be seen as the first presentation. $17.4 \%$ of patients with non-small cell lung carcinoma presented with leptomeningeal metastasis at the time of initial diagnosis [2]. Our case is an example where neurological symptoms are the first and only presentation of non-small cell lung adenocarcinoma. To our knowledge, this is the only case presenting with sensorial problems as the first symptom in lung adenocarcinoma in the literature.

For diagnosis, entire neuraxis magnetic resonance imaging including brain and spinal cord and cytological analysis of CSF to identify malignant cells are required. MRI with gadolinium enhancement may show subarachnoid, ventricular or parenchymal enhancing nodules, which are the most frequent MRI findings, focal or diffuse pial enhancement, ependymal, sulcal folia or cranial nerve enhancement as demonstrated in our case $[1,4]$. 
Citation: Guler S, Selcuk HH, Eren F, Apaydin E, Soysal A (2018) Leptomeningeal Metastasis as the First Presentation of Lung Adenocarcinoma. J Neurol Neurophysiol 9: 475. doi:10.4172/2155-9562.1000475

Because the sensitivity of MRI varies, lumbar puncture should be performed. Abnormal findings in CSF include increased opening pressure, increased leukocytes, elevated protein and decreased glucose. Identification of malignant cells in CSF can be accepted as the most important diagnostic feature. To increase sensitivity, CSF sampling should be done in large volumes $(>10 \mathrm{~mL})$ and at least two times [1,5]. The sensitivity of initial lumbar puncture is $71 \%$, whereas it increases after two (86\%) and three samples (90\%) [4]. For this reason, we performed lumbar puncture for three times.

Thyroid transcription factor- 1 is a $38-\mathrm{kDa}$ nuclear protein and lineage-specific transcription factor, involved in morphogenesis, differentiation, and surfactant production of normal lung epithelial cells. TTF-1 is known as a valuable immunohistochemical marker in adenocarcinomas of the lung (60-90\%) and thyroid gland, but not expressed in other primary and metastatic adenocarcinomas $[7,8]$. TTF-1 has a dual role in non-small cell lung adenocarcinoma as both tumor suppression and oncogenesis and believed to be a prognostic factor [7,9]. In our case, we found TTF-1 expression in malignant cells in both CSF and lung biopsy specimen [10].

\section{Conclusion}

Leptomeningeal metastasis is typically seen and diagnosed in late stages of malignancies, that causes inefficient treatment and poor prognosis. The pathological examination of CSF in patients with leptomeningeal contrast enhancement, as routine CSF examination, may be helpful in early diagnosis.

\section{Author's contributions}

Sena Guler, MD, PhD Candidate, Bakırköy Prof. Dr. Mazhar Osman Training and Research Hospital for Psychiatric and Neurological Diseases, Neurology, Istanbul, Turkey, Role: Acquisition of data, final approval.

Hatem Hakan Selcuk, MD, Bakırköy Dr. Sadi Konuk Training and Research Hospital, Neuroradiology, Istanbul, Turkey, Role: Interpretation of CT and MR images.
Fulya Eren, MD, Bakırköy Prof. Dr. Mazhar Osman Training and Research Hospital for Psychiatric and Neurological Diseases, Neurology, Istanbul, Turkey, Role: Acquisition of data.

Evsen Apaydin, MD, Istanbul University, Istanbul Faculty of Medicine Department of Pathology, Istanbul, Turkey, Role: Interpretation of microscopic images.

Aysun Soysal, MD, Bakırköy Prof. Dr. Mazhar Osman Training and Research Hospital for Psychiatric and Neurological Diseases, Neurology, Istanbul, Turkey, Role: Acquisition of data, final approval.

\section{References}

1. Rhun EL, Taillibert S, Chamberlain MC (2013) Carcinomatous meningitis: Leptomeningeal metastases in solid tumors. Surg Neurol Int 4: 265-288.

2. Lee S (2013) Leptomeningeal carcinomatosis in non-small-cell lung cancer patients, impact on survival and correlated prognostic factors. J Thorac Oncol 8: 185-191.

3. Hamouri S, Shorafat DA (2016) Bilateral facial nerve paralysis as first presentation of lung cancer. Case Rep Oncol 9: 792-795.

4. Clarke $\mathrm{JL}$ (2012) Leptomeningeal metastasis from systemic cancer. Continuum 2: 328-242.

5. Choi E, Lewis AL, Takei H, Ro JY (2012) Leptomeningeal carcinomatosis as initial presentation in adenocarcinoma of lung with signet ring cell features: An autopsy case report. Int J Clin Exp Pathol 5: 972-976.

6. Li H (2017) Applicability of graded prognostic assessment of lung cancer using molecular markers to lung adenocarcinoma patients with brain metastases. Oncotarget 8: 70727-70735.

7. Schilsky JB (2017) Prognostic impact of TTF-1 expression in patients with stage IV lung adenocarcinomas. Lung Cancer 108: 205-211.

8. Konno H (2017) Rapid immunohistochemistry with thyroid transcription factor-1 for pulmonary adenocarcinoma. Ann Thorac Surg 104: 471-476.

9. Huang TW, Lin KF, Lee $\mathrm{CH}$, Chang H, Lee SC, et al. (2017) The role of thyroid transcription Factor-1 and tumor differentiation in resected lung adenocarcinoma. Sci Rep 7: 14222.

10. Yserbyt J, Wilms G, Lievens Y, Nackaerts K (2009) A 63-year-old man with Peripheral Facial Nerve Paralysis and a Pulmonary Lesion. Acta Clinica Belgica 64-73. 